\title{
Effect of Ipriflavone on Osteoblasts and Osteoclasts during Guided Bone Augmentation
}

\author{
Motohiko Yagi, ${ }^{* a}$ Yoichi Ono, ${ }^{b}$ Tadashi Minegishi, ${ }^{b}$ Toshio Uchiyama, ${ }^{b}$ Kenji Tanaka, ${ }^{b}$ \\ Yuichi Mizumura, ${ }^{b}$ Shuichi Sato, ${ }^{b, c}$ and Koichi Ito ${ }^{b, c}$
}

${ }^{a}$ Division of Applied Oral Sciences, Nihon Universiuty Graduate School of Dentistry, ${ }^{b}$ Department of Periodontology, Nihon University School of Dentistry, and ${ }^{c}$ Division of Advanced Dental Treatment, Dental Research Center, Nihon University School of Dentistry, 1-813, Kanda Surugadai, Chiyoda-ku, Tokyo 101-8310, Japan

(Received March 12, 2007; Accepted May 23, 2007; Published online May 28, 2007)

\begin{abstract}
We investigated the effect of ipriflavone (IP) on osteoblasts and osteoclasts during guided bone augmentation (GBA). Each of ten rabbits had two titanium caps placed into its calvarium for GBA. The animals were divided into two groups: No-IP and IP (IP orally, $10 \mathrm{mg} / \mathrm{kg}$ daily after GBA). One cap was removed from each rabbit after 3 months, and the remaining cap served as a control. One month after the removal, all of the animals were euthanized, and histologic and histomorphometric analyses were performed. The tissue generated at the test site in the two groups was resorbed, and its original shape and volume were not maintained 1 month after cap removal. In particular, roughly $20 \%$ of the newly generated tissue in the No-IP group was resorbed $(72.9 \pm 2.6 \%$ vs. $92.4 \pm 3.3 \%$, $p<0.05$ ), while approximately $8 \%$ was resorbed in the IP group. Furthermore, more osteoblast-like cells were present in the IP group than in the No-IP group $(44.4 \pm 3.05 v s .30 .2 \pm 3.11, p<0.05)$, and the proportion of osteoclast-like cells was reduced in the IP group compared to the No-IP group $(0.59 \pm 0.02 v s .0 .76 \pm 0.06, p<0.05)$. Therefore, the amount of mineralized tissue generated appeared to increase as the total IP dose increased. Within the limitations of this experimental model, we conclude that daily intake of IP after GBA inhibits the resorption of the augmented tissue and may be useful for improving the quality of newly generated bone beyond the skeletal envelope.
\end{abstract}

Key words — bone augmentation, calvarial bone, guided bone augmentation, ipriflavone, rabbit

\section{INTRODUCTION}

Ipriflavone (IP), an isoflavone derivative, is used to treat osteoporosis by enhancing the retention of calcium. ${ }^{1)}$ IP inhibits osteoclast differentiation and activity and may promote bone formation during bone remodeling. ${ }^{2-5}$ )

It was recently shown that IP facilitates osteoblast activity and that IP and its metabolites facilitate cell proliferation, alkaline phosphatase activity, and collagen synthesis in a human osteosarcoma cell line. ${ }^{6)}$ Furthermore, IP is effective in reducing the bone turnover rate, mainly by inhibiting bone resorption, ${ }^{3,6,7)}$ but also by stimulating bone formation. ${ }^{8,9)}$ Therefore, IP appears to have several mechanisms of action, all of which enhance bone density;

\footnotetext{
${ }^{*}$ To whom correspondence should be addressed: Division of Applied Oral Sciences, Nihon Universiuty Graduate School of Dentistry, 1-8-13, Kanda Surugadai, Chiyoda-ku, Tokyo 1018310, Japan. Tel.: +81-3-3219-8107; Fax: +81-3-3219-8349; E-mail: yagi@dent.nihon-u.ac.jp
}

in particular, it possesses bone-forming properties and is considered to be anti-resorptive. Studies on the effects of IP on perialveolar bone formation suggest that IP is a potential therapeutic tool for promoting the repair of injured alveolar bone. ${ }^{10)}$ In addition, in humans, it has also been suggested that IP prevents bone loss ${ }^{1-14)}$ and increases bone mass in postmenopausal women. ${ }^{15-17)}$

However, insufficient histomorphometric data exist regarding the in vivo effect of IP on bone formation beyond the skeletal envelope during guided bone augmentation (GBA). In addition, it is unclear whether IP prevents the resorption of augmented tissue and whether it promotes bone mineralization in tissue generated by GBA.

Therefore, we investigated the potential pharmacological effect of IP on osteoblasts and osteoclasts in augmented bone during GBA. 


\section{MATERIALS AND METHODS}

Animals and Surgical Procedures — Ten adult male Japanese white rabbits, each weighing 2.4$3.1 \mathrm{~kg}$, were used. The methods used in this study were approved by the Animal Experimentation Committee at Nihon University School of Dentistry.

General anesthesia was induced by inhalation of halothane gas $\left(1.5-2 \%\right.$, vol\%; Fluothane ${ }^{\circledR}$, Takeda Chemical Industries, Ltd., Osaka, Japan) and by injection of pentobarbital sodium $(0.4 \mathrm{ml} / \mathrm{kg}$; Nembutal ${ }^{\circledR}$, Abbot Laboratories, North Chicago, IL, U.S.A.) via an ear vein. In addition, approximately $1 \mathrm{ml}$ of local anesthetic with $\mathrm{HCl}$ containing epinephrine at 1:80000 $\left(2 \%\right.$ Xylocain ${ }^{\circledR}$, Astra Japan, Fujisawa Pharmaceutical Co., Osaka, Japan) was used to reduce hemorrhaging. After the forehead of each rabbit was shaved, a cutaneous flap was created and lifted to expose the calvarial bone on both sides of the midline.

The experimental device was a custom-made, standardized stiff titanium hemispherical cap $(\mathrm{Ti}>$ 99.5\%; JIS H 6400, Sankin, Tokyo, Japan) with a smooth surface. The cap was $4 \mathrm{~mm}$ in height, $8 \mathrm{~mm}$ in diameter, and $0.2 \mathrm{~mm}$ thick. A circular groove with an inner diameter of $8 \mathrm{~mm}$ was prepared in the bone on each side of the midline using a trephine drill (Bone trephine 131001, Technica, Tokyo, Japan). Then, nine small holes were drilled with a number two round bur to induce bleeding from the marrow space, and a standardized titanium cap was placed in the circular groove by press-fit. The rabbits were randomly assigned to either the No-IP (no intake of IP) or IP $(10 \mathrm{mg} / \mathrm{kg}$ IP once a daily orally after GBA for 4 months) group. IP was prepared at $10 \mathrm{mg} / \mathrm{ml}$ using a $1 \%$ hydroxypropyl cellulose solution.

Each flap was repositioned to cover the titanium cap and sutured. Postoperatively, the animals received antibiotic therapy $(2500000 \mathrm{IU} / \mathrm{ml}$ penicillin G, Yamanouchi, Tokyo, Japan) at a dose of $0.1 \mathrm{ml} / \mathrm{kg}$, given as a single intramuscular injection. Furthermore, to investigate the inhibitory effect of IP on the absorption of the newly generated tissue and on the osteoblasts and osteoclasts, after 3 months, a single titanium cap was removed from each rabbit under general anesthesia as described above. This site was defined as the test site, and the site of the remaining cap was defined as the control site [Fig. 1(A) and (B)].
(A)

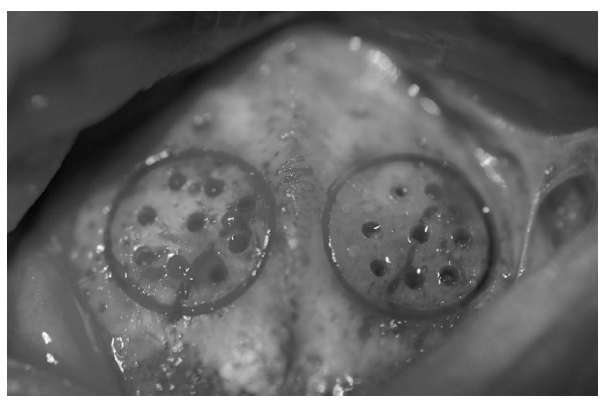

(B)

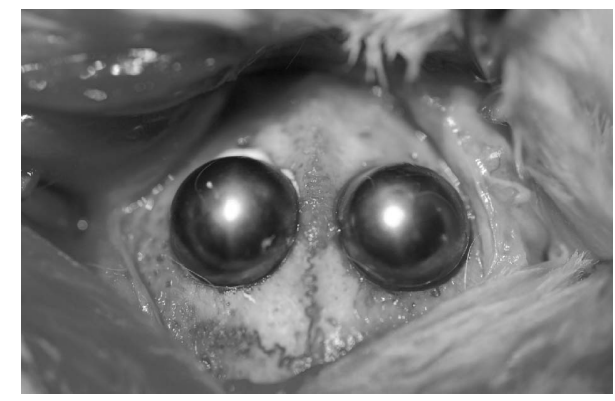

Fig. 1. A Cutaneous Flap was Demarcated (A) and a Titanium Cap was Placed in the Bone so That the Margin of the Cap Fit Neatly Into the Groove (B)

Specimen Preparation — One month after cap removal, the animals were euthanized with an overdose of pentobarbital. The calvaria were dissected, fixed in $10 \%$ neutral buffered formaline, dehydrated, and embedded in a polyester resin (Rigolac2004, Rigolac-70F, Nisshin EM, Tokyo, Japan). One sagittal undecalcified ground section (approximately $200 \mu \mathrm{m}$ thick) was prepared from the midportion using a low-speed diamond saw (Micro Cutter, MC-201, Maruto, Tokyo, Japan).

Histological and Histomorphometric Analyses_- Histological examination, photography, and histomorphometric assessment of the sections were performed as reported elsewhere. ${ }^{25)}$ The histomorphometric data obtained from each specimen were recorded with a computerized image analysis system (Adobe Photoshop ${ }^{\circledR}, 4.01$ J, Adobe Systems, Tokyo, Japan). Slides taken at $\times 3$ magnification were digitized using a solid-state, $35-\mathrm{mm}$ slide scanner and a charge coupled device (CCD) linear photo diode array interfaced with a computer.

The percent area of newly generated tissue consisting of mineralized bone and marrow space in each histological section of the control site was calculated relative to the area bounded by the hemispherical shape of the titanium cap and the host bone; this latter volume was taken as $100 \%$. In addition, we determined the cross-sectional area of the newly mineralized bone expressed as the percentage 


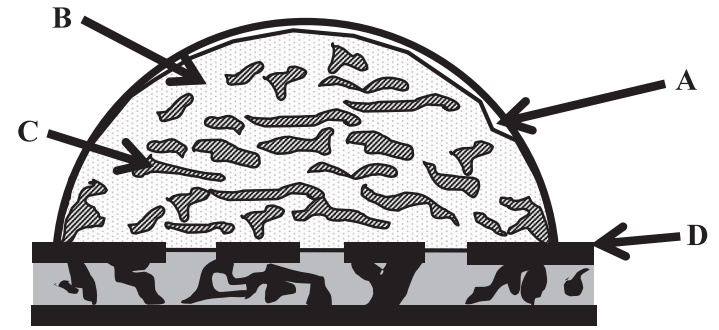

Fig. 2. Schematic of a Sagittal Histological Section Through the Center of a Titanium Cap Illustrating the Measurements Performed

(A): area of the titanium cap, (B): area of newly generated tissue expressed as the percentage of (A), (C): area of newly generated mineralized bone expressed as the percentage of (B). Note that (D): the baseline used to imitate the cross-sectional contour of the original parent bone.

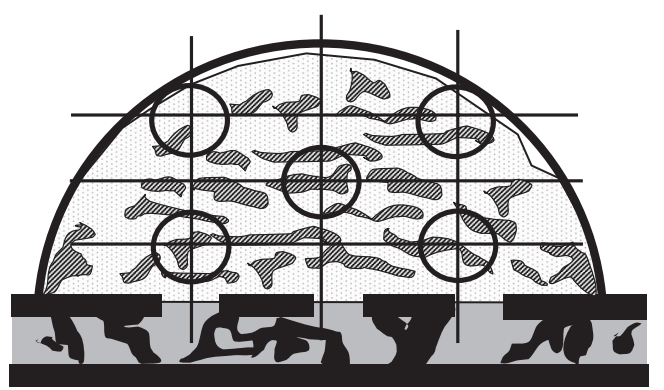

Fig. 3. The Mean Numbers of Osteoblast- and Osteoclastlike Cells were Measured Manually under Light Microscopy at $\times 200$ Magnification

Five random fields were examined for each section.

of the total tissue area generated within each space. At the test site (i.e., where one cap was removed), a hypothetical cap with the same shape and size as the original was computer-generated in the histological sections (semicircular line, Fig. 2).

Cell Count — The mean numbers of osteoblastand osteoclast-like cells were determined manually under light microscopy at $\times 200$ magnification. In total, five standardized fields were examined for each section (Fig. 3).

Statistical Analysis — All of the data are presented as the means \pm S.D. The data were analyzed by the Mann-Whitney $U$-test (intergroup comparison) and Wilcoxon's test (intragroup comparison). Differences at $p<0.05$ were considered significant. The statistical program SPSS ${ }^{\circledR}$ Base 10.0J (SPSS Japan, Tokyo, Japan) was used for all analyses.

\section{RESULTS}

\section{Histological Observation}

Healing was uneventful at all of the surgical sites in all of the rabbits; consequently, five specimens were used for each control and test site in the No-IP and IP groups. During specimen retrieval and dissection, which involved removing the cutaneous layer overlaying the caps, no signs of inflammation, pathology, or adverse reaction were noted at the surgical sites. In the control groups, the titanium caps were tightly placed in the host bone. In all of the groups, the newly generated tissue was of varying size; it consisted of thin pieces of mineralized bone and large marrow spaces with fat cells and some hematopoietic cells underneath the titanium cap or the hypothetical cap. No connective tissue was present under the caps. The generated bone layer tended to climb along the inner wall of the cap in close contact with the titanium surface and covered the generated tissues [Fig. 4(A)-(D)]. Cuboidal osteoblast-like cells were found to be actively laying down bone to varying degrees along the mineralized bone in both groups. Osteoblastlike cells were more common in the IP group than in the No-IP group [Fig. 5(A)-(D)].

\section{Histomorphometric Evaluation}

The Mann-Whitney $U$-test revealed a significant $(p<0.05)$ difference in the newly generated tissue at the control and test sites in the No-IP group; however, a significant $(p<0.05)$ difference was not detected at the control and test sites in the IP group. Newly generated tissue was observed at all of the control sites, almost filling the space under the cap.

No significant difference was observed in the amount of tissue generated between the No-IP and IP groups $(92.4 \pm 3.3 \%$ vs. $94.6 \pm 7.3 \%)$. Furthermore, the tissue generated at the test sites in the two groups was resorbed, and its original shape and volume were not maintained 1 month after the removal of a single cap; reductions of approximately $20 \%$ and $8 \%$ were found in the No-IP and IP groups, respectively. In the No-IP group, a significantly greater amount of new tissue formed at the control site compared to the test site $(p<0.05)$ (Table 1$)$. The relative amount of mineralized bone generated at the control and test sites was significantly larger in the IP group than in the No-IP group $(p<0.05)$. Furthermore, a significant difference was detected in the relative amount of mineralized bone generated between the control and test sites in the No-IP 
(A)

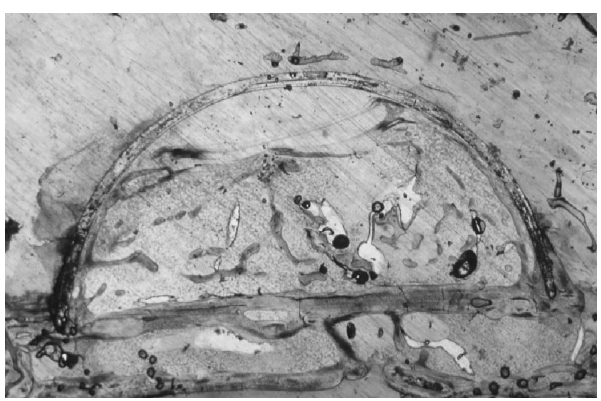

(C)

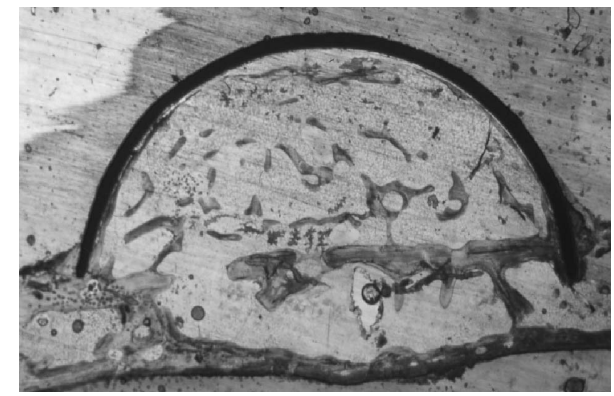

(B)

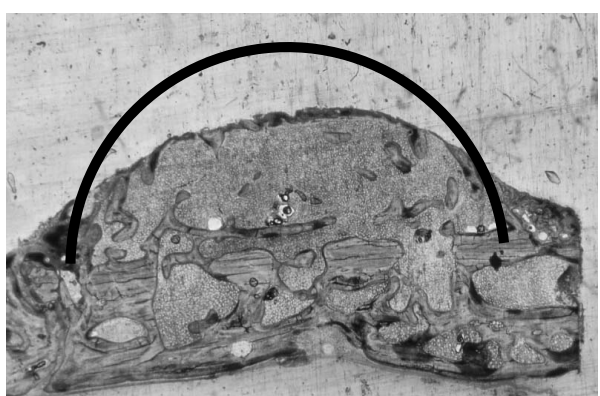

(D)

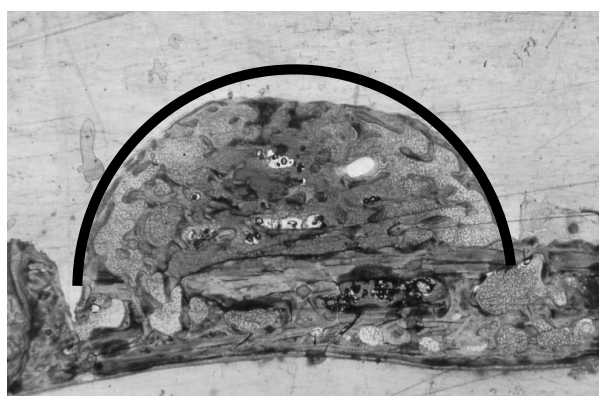

Fig. 4. Typical Sagittal Histological Sections of the Control (A) and Test (B) Sites in the No-IP Group. Tissue with No Signs of Connective Tissue Grew under the Cap to Various Degrees. A Slender Layer of Mineralized Bone Climbed Along the Inner Wall of the Cap and Covered the Generated Tissue. A Titanium Cap was Removed 1 Month before the Animals were Euthanized. Some Resorption of the Newly Generated Tissue was Observed Compared to the Control Site. Typical Sagittal Histological Sections of the Control (C) and Test (D) Sites in the IP Group

New tissue grew under the cap, almost filling the space at the control site. No obvious differences in the appearance of the newly generated tissue were observed, other than a clear reduction in the amount of tissue at the test site. Sagittal histological section of the titanium cap at the control site in the No-IP group. A hypothetical titanium cap with the same dimensions as the original cap in (B) and (D) is outlined in black. The sections were stained with basic fuchsin and methylene blue. Original magnification, $\times 3$.

(A)

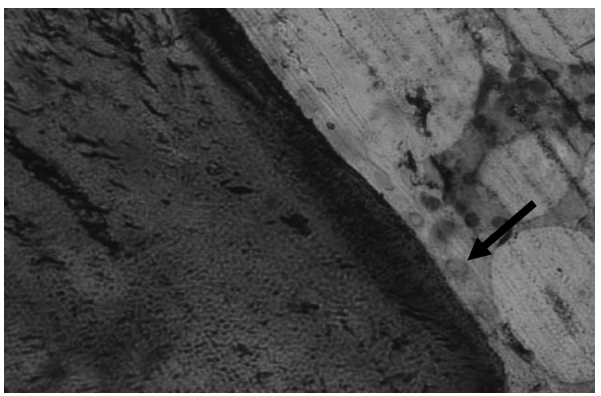

(C)

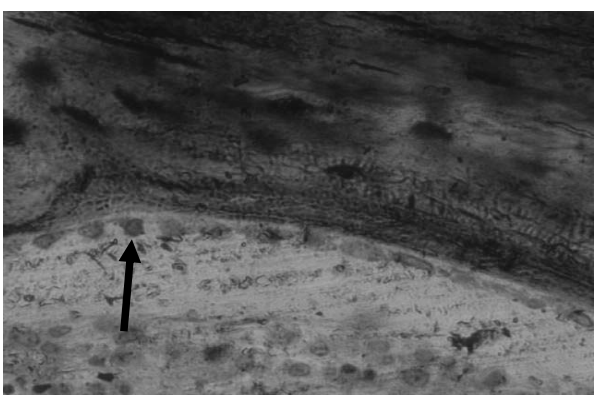

(B)

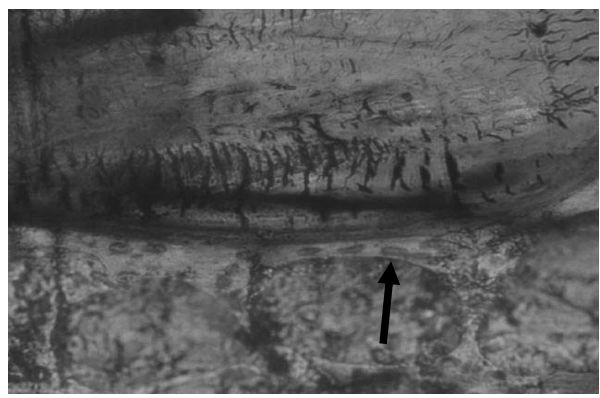

(D)

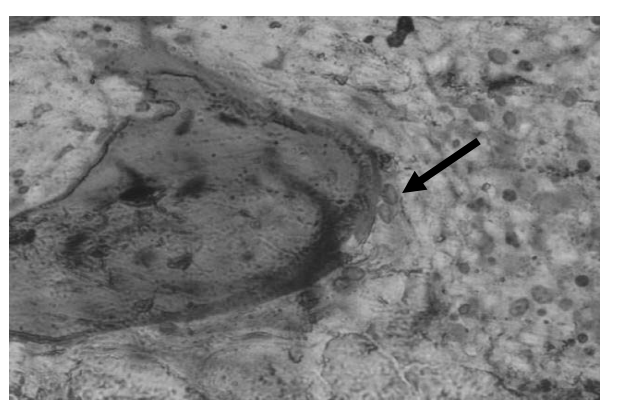

Fig. 5. Typical Histological Sections of the Control Sites in the (A) No-IP and (C) IP Groups. Generally, Cuboidal Osteoblast-like Cells (arrow) Actively Laid Down Bone on the Mineralized Bone at the Control Sites in All of the Groups. A Similar Amount of Osteoblastic Activity was Observed at the Test Site in the (B) No-IP and (D) IP Groups

The sections were stained with basic fuchsin and methylene blue. Original magnification, $\times 200$. 
Table 1. The Parcentage Areas of Newly Generated Tissue Under the Titanium Cap

\begin{tabular}{|c|c|c|c|c|}
\hline group & $\mathrm{N}$ & control & \multicolumn{2}{|l|}{ test } \\
\hline & & \multicolumn{3}{|c|}{$\dagger$} \\
\hline No-IP & 5 & $92.4 \pm 3.3$ & $72.9 \pm 2.6$ & \\
\hline IP & 5 & $94.6 \pm 7.3$ & $86.3 \pm 2.2$ & \\
\hline
\end{tabular}

Mean S.D., unit. $\%{ }^{*} p<0.05$, analysed by the Mann-Whitney. ${ }^{\dagger} p<0.05$, analysed by the Wilcoxon test. IP: ipriflavone, No-IP: no intake of IP, IP: $10 \mathrm{mg} / \mathrm{kg}$ IP daily orally after guided bone augmentation (GBA)

Table 2. The Parecentage Area of Mineralized Bone in the Newly Generated Tissue Under Titanium Cap

\begin{tabular}{|c|c|c|c|c|c|}
\hline group & $\mathrm{N}$ & control & & test & \\
\hline & & \multicolumn{4}{|c|}{$\dagger$} \\
\hline No-IP & 5 & $21.7 \pm 1.1$ & & $27.3 \pm 1.9$ & \\
\hline IP & 5 & $34.8 \pm 3.8$ & & $33.3 \pm 2.1$ & \\
\hline
\end{tabular}

Mean S.D., unit.\%. ${ }^{*} p<0.05$, analysed by the Mann-Whitney test. ${ }^{\dagger} p<0.05$, analysed by the Wilcoxon test. IP: ipriflavone, NoIP: no intake of IP, IP: $10 \mathrm{mg} / \mathrm{kg}$ IP daily orally after guided bone augmentation (GBA).

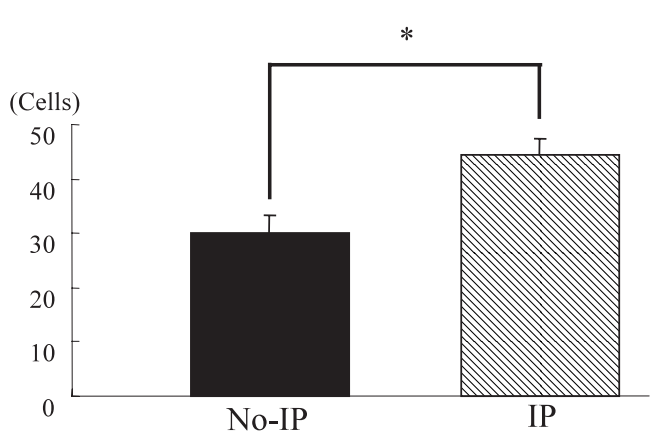

Fig. 6. Number of Osteoblast-like Cells in the Newly Generated Tissue

The number of osteoblast-like cells present in the IP group was significantly higher than in the No-IP group $(44.4 \pm 3.05$ vs. $30.2 \pm$ $3.11, p<0.05$ analysed by the Mann-Whitney test).

group $(p<0.05)$ (Table 2).

\section{Numbers of Osteoblast- and Osteoclast-like Cells}

After 4 months of healing, significantly more osteoblast-like cells were observed in the IP group than in the No-IP group $(44.4 \pm 3.05$ vs. $30.2 \pm 3.11$, $p<0.05$ ) (Fig. 6). Similarly, after 4 months of healing, significantly fewer osteoclast-like cells occurred in the IP group compared to the No-IP group $(0.59 \pm 0.02$ vs. $0.76 \pm 0.06, p<0.05)$ [Figs. 7 and

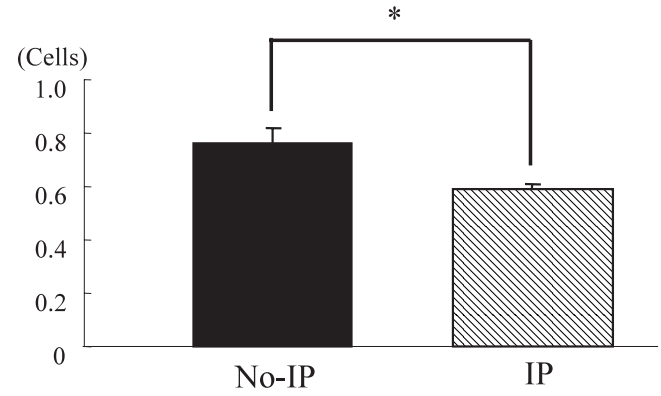

Fig. 7. Number of Osteoclast-like Cells in the Newly Generated Tissue

The number of osteoclast-like cells was significantly reduced in the IP group compared to the No-IP group $(0.59 \pm 0.02$ vs. $0.76 \pm 0.06$, $p<0.05$ analysed by the Mann-Whitney test).

8(A)-(D)].

\section{DISCUSSION}

In this study, we demonstrated that by using a titanium cap it is possible to augment the generation of bone beyond the skeletal envelope into areas in which no bone was previously present. This finding concurs with studies in which a titanium device (e.g., a cap, dome, or cylinder) was used to evaluate the potential for new bone formation. ${ }^{18-26)}$ Among the rabbits in this study, newly generated tissue occupied $92-99 \%$ of the total possible space created by the caps after 4 months of healing with or without IP. This is similar to the results (80-97\%) from several 3-month studies that used the same titanium cap model. ${ }^{20,22,23,25)}$

Our results demonstrate that the tissue generated on the surface of the rabbit calvaria by GBA is not stable over time. Some resorption, equivalent to between 8 and $20 \%$ of the newly generated tissue, was observed within the first month after the removal of a single titanium cap. This resorption can probably be ascribed to surgical trauma during cap removal, which exposed the newly generated tissue. A significant difference was detected in the resorption of newly generated tissue between the control and test sites in the No-IP group: approximately $20 \%$ of the newly generated tissue was resorbed $(92.4 \pm 3.3 \% v s .72 .9 \pm 2.6 \%, p<0.05)$. Therefore, IP may inhibit further resorption of the tissue generated by GBA. We believe that bone remodeling actively occurred in the tissue generated by GBA. Bone remodeling refers to the sequential, coupled actions of osteoclasts and osteoblasts. Osteoblasts stimulate bone formation and calcification 
(A)

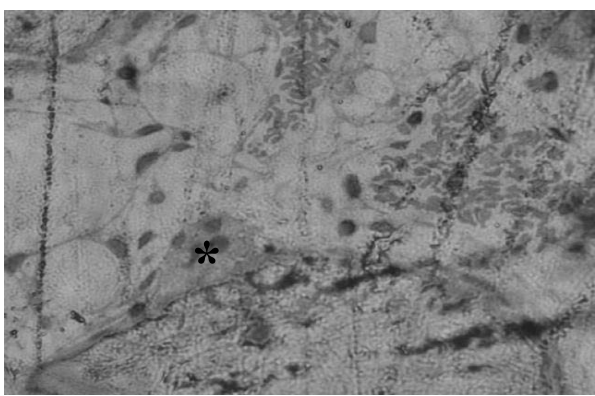

(C)

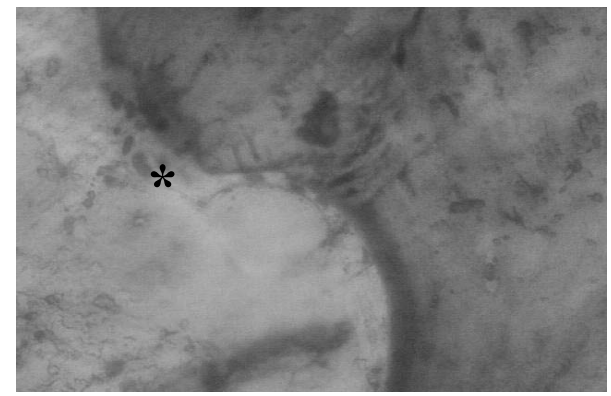

(B)

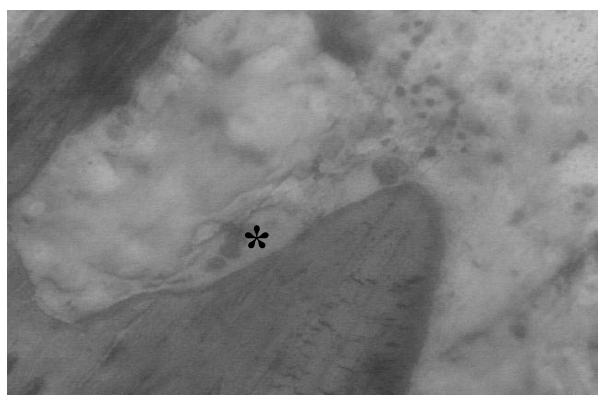

(D)

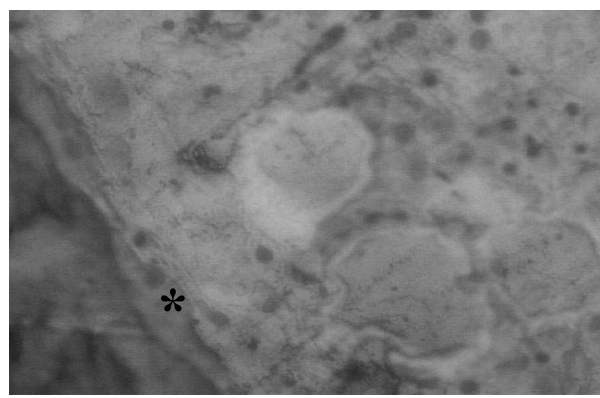

Fig. 8. Typical Histological Sections of the Control Sites in the (A) No-IP and (C) IP Groups and of the Test Sites in the (B) No-IP and (D) IP Groups

Osteoclast-like cells $\left(^{*}\right)$ were reduced in the IP group compared to the No-IP group. The sections were stained with basic fuchsin and methylene blue. Original magnification, $\times 200$.

while osteoclasts promote bone resorption. This cyclical process normally maintains the status quo and does not change the size or shape of bones. Bone remodeling removes a portion of old bone and replaces it with new bone. Thus, we determined the numbers of osteoclast- and osteoblast-like cells to investigate the effect of IP on these cell types.

After 4 months of healing, the number of osteoblast-like cells in the newly generated tissue was $30.2 \pm 3.11$ in the No-IP group and $44.4 \pm 3.05$ in the IP group. Moreover, the number of osteoclastlike cells in the newly generated tissue was $0.76 \pm$ 0.06 in the No-IP group and $0.59 \pm 0.02$ in the IP group. More osteoblast-like cells were present in the IP group than in the No-IP group. In addition, the proportion of osteoclast-like cells decreased in the IP group compared to the No-IP group. However, significant numbers of osteoblast-like cells and osteoclast-like cells were not observed at the control and test sites in the IP and No-IP groups. IP appears to exhibit anti-resorptive and bone-forming properties. Its anti-resorptive effect involves inhibiting the activation of mature osteoclasts and the formation of new osteoblasts. ${ }^{3,6)}$

In contrast, the osteoblastic effect of IP and its metabolites stimulated the proliferation of an osteoblast-like cell line (UMR-106a), increased alkaline phosphatase activity, and enhanced colla- gen formation. ${ }^{8)}$ Furthermore, Kawamoto (2000) investigated the effects of IP on cell growth, alkaline phosphatase activity, mineralized nodule formation, gene expression of growth factors and their receptors, and extracellular matrix using a human osteosarcoma cell line (Saos-2). Alkaline phosphatase activity was elevated by IP. The expression of transforming growth factor (TGF)- $\beta 1$, insulinlike growth factors (IGF)-1, and bone morphogenetic protein (BMP)-2 were stimulated by IP, but no effect occurred in their receptors. Expression of the platelet-derived growth factor (PDGF) $\beta$ and PDGF- $\beta$ receptors was increased by IP. The expression of bone sialoprotein and osteonectin was stimulated by IP, but no effect was observed on type I collagen. These results indicate that IP may promote bone formation during bone remodeling due to increased growth factor expression. ${ }^{27)}$ Bone marrow osteoprogenitor cells and trabecular bone osteoblasts were isolated from human donors and incubated with IP and its metabolites. These substances were found to regulate osteoblast differentiation by enhancing the expression of important bone-matrix proteins and by facilitating mineralization. ${ }^{9)}$

Martini et al. (1998) provided further evidence of the direct effect of IP on osteoblast activity. A small, circular cavity ( $3 \mathrm{~mm}$ in diameter) was sur- 
gically created in rat mandible and filled with powdered IP. Local application of IP is recommended to enhance and accelerate bone formation in the repair of a surgically created hole. They concluded that IP stimulates osteogenesis and is a potential therapeutic tool for promoting the repair of injured perialveolar bone.

Minegishi et al. (2002) administered a single 400-mg dose of IP during GBA in a rabbit calvarial model. They suggested that IP affects the quality of bone augmentation at an early stage. Light microscopic examination of test sites packed with IP in a collagen gel showed that mineralized bone formed that surrounded the IP. Furthermore, IP was effective in reducing the bone turnover rate, primarily by stimulating bone formation and possibly by inhibiting bone resorption. Although IP appeared to be resorbed gradually, it was not completely resorbed 3 months after surgery. Therefore, residual IP in the titanium cap may inhibit further bone formation within a limited space. These findings are consistent with those using bone graft materials, such as BIOOSS $^{\circledR}$ and $\beta$-tricalcium phosphate residue, in the titanium cap, ${ }^{20,22)}$ and are also in agreement with the observations of other studies using biomaterials.

To assess the potential impact of IP on newly generated tissue and bone mineralization during GBA, a daily oral dose $(10 \mathrm{mg} / \mathrm{kg})$ of IP was administered to rabbits for 4 months. Generally, $200 \mathrm{mg}$ of IP is administered orally three times daily in osteoporosis therapy, ${ }^{12-15)}$ which is equivalent to an IP dose of $10 \mathrm{mg} / \mathrm{kg}$ for an average 60-kg human. Therefore, the daily dose of IP used in this study $(10 \mathrm{mg} / \mathrm{kg})$ is reasonable when compared to the human therapeutic dose.

One must consider whether the bone tissue generated beyond the skeletal envelope will be maintained on a long-term basis after removing the titanium cap. In our study, some bone remodeling occurred after cap removal in the newly generated tissue under the titanium caps; the amount of new tissue remaining at the test site 1 month after cap removal differed from that at the control site.

Whether the relatively slender pieces of bone that formed in our titanium cap model will become denser after further healing or will gradually resorb remains to be determined. A significant acrossgroup difference was detected in the percentage of mineralized bone in the newly generated tissue at the control sites. The percentage of mineralized bone in each group gradually increased with the total dose of IP (No-IP: 22\%, IP: 35\%). Furthermore, at the test sites, the percentage of mineralized bone in the IP group was $33 \%$. In addition, the percentage of mineralized bone at both the test and control sites in the IP group was greater than previously reported percentages. These findings indicate that daily administration of IP may potentially improve the quality of bone generated by GBA. The quality of newly generated bone and the percentage of mineralized bone may depend on the total dose of IP.

Within the limitations of our experimental model, we conclude that daily intake of IP after GBA inhibits the resorption of augmented tissue and may be useful for improving the quality of newly generated bone beyond the skeletal envelope.

Acknowledgements We thank Textcheck for excellent assistance in preparing this manuscript. Thanks are also due to Takeda Chemical Industries, Ltd., Osaka, Japan, for the donation of IP. This study was supported in part by a 1999 grant from the Sato Fund, Nihon University School of Dentistry, and by a 2000-2002 Grant-in-Aid for Scientific Research (C-2, \#12672042) from the Ministry of Education, Culture, Sports, Science and Technology, Japan.

\section{REFERENCES}

1) Gennari, C. (1997) Ipriflavone: background. Calcif. Tissue Int., 61 (Suppl. 1), 3-4.

2) Bonucci, E., Silvestrini, G., Ballanti, P., Masi, L., Franchi, A., Bufalino, L. and Brandi, M.L. (1992) Cytological and ultrastructural investigation on osteoblastic and preosteoclastic cells grown in vitro in the presence of ipriflavone: preliminary results. Bone Miner. 19 (Suppl. 1), 15-25.

3) Bonucci, E., Ballanti, P., Martelli, A., Mereto, E., Brambilla, G., Bianco, P. and Bufalino, L. (1992) Ipriflavone inhibits osteoclast differentiation in parathyroid transplanted parietal bone of rats. Calcif. Tissue Int., 50, 314-319.

4) Sziklai, I. and Ribari, O. (1985) The effect of flavone treatment on human otosclerotic ossicle organ cultures. Arch. Otorhinolaryngol., 242, 67-70.

5) Ribari, O. and Sziklai, I. (1987) Effect of flavonoid on RGE2-induced alterations in percentage collagen synthesis in ossicle organ cultures. Acta Otolaryngol., 103, 657-660.

6) Notoya, K., Yoshida, K., Taketomi, S., Yamazaki, I. and Kumegawa, M. (1993) Inhibitory effect of ipriflavone on osteoclast-mediated bone resorption and new osteoclast formation in long-term cultures of 
mouse unfractionated bone cells. Calcif. Tissue Int., 53, 206-209.

7) Albanese, C. V., Cudd, A., Argentino, L., ZamboninZallone, A. and MacIntyre, I. (1994) Ipriflavone directly inhibits osteoclastic activity. Biochem. Biophys. Res. Commun., 199, 930-936.

8) Benvenuti, S., Tanini, A., Frediani, U., Bianchi, S., Masi, L., Casano, R., Bufalino, L., Serio, M. and Brandi, M. L. (1991) Effect of ipriflavone and its metabolites on a clonal osteoblastic cell line. J. Bone Miner. Res., 6, 987-996.

9) Cheng, S. L., Zhang, S. F., Nelson, T. W., Warlow, P. M. and Civitelli, R. (1994) Stimulation of human osteoblast differentiation and function by ipriflavone and its metabolites. Calcif. Tissue Int., 55, 356-362.

10) Martini, M., Formigli, L., Tonelli, P., Giannelli, M., Amunni, F., Naldi, D., Brandi, M. L., Zecchi Orlandini, S. and Orlandini, G. E. (1998) Effects of ipriflavone on perialveolar bone formation. Calcif. Tissue Int., 63, 312-319.

11) Agnusdei, D., Zacchei, F., Bigazzi, S., Cepollaro, C., Nardi, P., Montagnani, M. and Gennari, C. (1989) Metabolic and clinical effects of ipriflavone in established post-menopausal osteoporosis. Drugs Exp. Clin. Res., 15, 97-104.

12) Adami, S., Bufalino, L., Cervetti, R., Di Marco, C., Di Munno, O., Fantasia, L., Isaia, G. C., Serni, U., Vecchiet, L. and Passeri, M. (1997) Ipriflavone prevents radial bone loss in postmenopausal women with low bone mass over 2 years. Osteoporos Int., 7, 119-125.

13) Gennari, C., Agnusdei, D., Crepaldi, G., Isaia, G., Mazzuoli, G., Ortolani, S., Bufalino, L. and Passeri, M. (1998) Effect of ipriflavone-a synthetic derivative of natural isoflavones - on bone mass loss in the early years after menopause. Menopause, 5, 9-15.

14) Ohta, H., Komukai, S., Makita, K., Masuzawa, T. and Nozawa, S. (1999) Effects of 1-year ipriflavone treatment on lumbar bone mineral density and bone metabolic markers in postmenopausal women with low bone mass. Horm. Res., 51, 178-183.

15) Passeri, M., Biondi, M., Costi, D., Bufalino, L., Castiglione, G. N., Di Peppe, C. and Abate, G. (1992) Effect of ipriflavone on bone mass in elderly osteoporotic women. Bone Miner., 19 (Suppl. 1), S57-S62.

16) Moscarini, M., Patacchiola, F., Spacca, G., Palermo, P., Caserta, D. and Valenti, M. (1994) New perspectives in the treatment of postmenopausal osteoporosis: ipriflavone. Gynecol. Endocrinol., 8, 203-207.
17) Agnusdei, D., Crepaldi, G., Isaia, G., Mazzuoli, G., Ortolani, S., Passeri, M., Bufalino, L. and Gennari, C. (1997) A double blind, placebo-controlled trial of ipriflavone for prevention of postmenopausal spinal bone loss. Calcif. Tissue Int., 61, 142-147.

18) Schmid, J., Hämmerle, C. H. F., Olah, A. J. and Lang, N. P. (1994) Membrane permeability is unnecessary for guided generation of new bone. An experimental study in the rabbit. Clin. Oral Implants Res., 5, 125-130.

19) Majzoub, Z., Berengo, M., Giardino, R., Aldini, N. N. and Cordioli, G. (1999) Role of intramarrow penetration in osseous repair: a pilot study in the rabbit calvalia. J. Periodontol., 70, 1501-1510.

20) Nanba, K. (1999) Effects of bone graftmaterial (BIOOSSs) on bone augmentation within the titanium cap in rabbit parietal bone. Nihon University Dental Journal, 73, 480-487.

21) Lundgren, A. K., Lundgren, D., Hämmerle, C. H. F., Nyman, S. and Sennerby, L. (2000) Influence of decortication of the donor bone on guided bone augmentation: an experimental study in the rabbit skull bone. Clin. Oral Implants Res., 11, 99-106.

22) Takaoka, K. (2001) Effects of a-tricalciumphosphate on bone augmentation within the titanium cap in rabbit parietal bone. The Japanese Journal of the Conservative Dentistry, 44, 115-123.

23) Minegishi, T., Kawamoto, K., Yamada, Y., Oshikawa, M., Kishida, M., Sato, S. and Ito, K. (2002) Effects of ipriflavone on bone augmentation within a titanium cap in rabbit calvaria. J. Oral Sci., 44, 7-11.

24) Slotte, C. and Lundgren, D. (2002) Impact of cortical perforations of contiguous donor bone in a guided bone augmentation procedure: an experimental study in the rabbit skull. Clin. Oral Implants Res., 4, 1-10.

25) Yamada, Y., Nanba, K. and Ito, K. (2003) Effects of occlusiveness of a titanium cap on bone generation beyond the skeletal envelope in the rabbit calvarium. Clin. Oral Implants Res., 14, 455-463.

26) Tamura, T., Fukase, Y., Goke, E., Yamada, Y., Sato, S., Nishiyama, M. and Ito, K. (2005) Threedimensional evaluation for augmented bone using guided bone regeneration. J. Periodontal. Res., 40, 269-276.

27) Kawamoto, K. (2002) The effect of ipriflavone on the expression of growth factors and these receptors in human osteosarcoma cell line. Nihon University Dental Journal, 74, 364-373. 